\title{
Preface to BIT 53:4
}

\author{
Axel Ruhe
}

Published online: 14 November 2013

(C) Springer Science+Business Media Dordrecht 2013

This year, we wind up BIT with a selection of papers from our regular stream of contributions. I note that now we have quite a few manuscripts in the Approximation area, both geometric design and numerical cubature, as well as some contributions to Numerical Linear Algebra. Only one paper deals with properties of time stepping methods for differential equations. But rest assured, more is coming up next year!

These are the papers:

Assyr Abdulle, Gilles Vilmart, and Konstantinos Zygalakis investigate the stability properties of integrators for Itô stochastic differential equations. They derive a singly diagonally implicit Runge Kutta method of weak second order, and compares its stability region to existing variants. Mean square and asymptotic stability is considered, as well as different variants of A stability.

A.K.B. Chand and P. Viswanathan study shape preservation aspects of cubic Hermite fractal interpolation functions. They get a desirable shape by putting constraints on values of function and derivative at the knot points. Scaling factors in the subintervals are chosen, to force the function to stay inside a rectangle.

Raffaele D'Ambrosio, Ernst Hairer, and Christophe J. Zbinden study long term integration of a conservative dynamical system. They prove that G-symplecticity of a general linear method implies conjugate-symplecticity of the underlying one step method.

Catterina Dagnino, Paola Lamberti, and Sara Remogna construct new cubature rules for 3D integrals, based on spline quasi-interpolants, expressed as linear combinations of scaled and translated boxes and local linear functionals. Nodes and weights are given, as well as error bounds.

A. Ruhe $(\otimes)$

Numerical Analysis Group, School of Science (SCI), Royal Institute of Technology (KTH), 10044, Stockholm, Sweden

e-mail: ruhe@kth.se 
Erik Martin-Dorel, Guillaume Melquiond, and Jean-Michel Muller study floating point computation in the practically interesting case where some parts of the computation are done in a higher precision than the original data. These computations are often a part of a much larger process, and then predictability is of importance, in order to get reliable bounds on the error imposed.

Ralf Hiptmair, Carlos Jerez-Hanckes, and Christoph Schwab study the Maxwell cavity source problem in the frequency domain. They use discretization by means of sparse tensor edge elements, in the case when a full tensor product gives rise to a basis of too high a dimension.

Tsung-Ming Huang, Zhongxiao Jia, and Wen-Wei Lin project a quadratic eigenvalue problem on a subspace, giving Ritz approximations to eigenvalues and eigenvectors. When the angle between an eigenvector and the subspace gets small, the Ritz values converge to the eigenvalue. They introduce the use of refined Ritz vectors which are proved to converge towards an eigenvector.

Zhongxiao Jia and Qian Zhang do a systematic study on how to choose drop tolerance to obtain a sparse approximative inverse, to be used in a preconditioned iterative algorithm for a nonsymmetric linear system.

Shishun Li and Zhengda Huang consider multigrid methods for nonselfadjoint elliptic problems, using a Hermitian/skew-Hermitian splitting as smoother. These iterations converge uniformly, even without a full elliptic regularity assumption, provided that the mesh size of the coarsest grid is sufficiently small, independent of the number of levels.

Marie-Laurence Mazure studies rational functions used for computer aided geometric design. They form an extended Chebyshev space that can be built up by a basis of quotients between Bernstein polynomials. This establishes a relation between Chebyshev spaces good for design and rational functions based on them, and offers new insights on rational Bézier curves.

Yujie Zhang and Yangfeng Su describe a two-level orthogonalization Arnoldi process to reduce a high dimensional delay system into a reduced order delay system. It needs less memory than the standard method, which is based on linear Arnoldi.

\section{Referees}

It is a recurring pleasure for me to acknowledge all of you that have helped us to keep the quality and readability of BIT. As is our custom, the referee of each paper is unknown to the author, yet we are all the ones that form, what we use to call the BIT family. Thank you all, editors, referees, and publishers for another good year with BIT!

These are the referees that I found in the files of BIT this year. Forgive me if I have missed someone that properly should deserve to be here:

\author{
Ben Adcock \\ Ravi P Agarwal \\ Pierluigi Amodio \\ Andreas Asheim \\ Winfried Auzinger
}

Gerard Awanou

Lori Badea

Zhong-Zhi Bai

Johnathan M Bardsley

Domingo Barrera 
Roland Becker

Bernhard Beckermann

John Belward

Sergio Blanes

Peter Blomgren

Paola Boito

Matthias Bollhöfer

J. P. Boyd

Richard P Brent

Evelyn Buckwar

Kevin Burrage

Pamela Burrage

Albrecht Böttcher

Marco Caliari

Felix Carbonell Gonzáles

Andrea Censi

Bilal Chanane

Philippe Chartier

Snorre H. Christiansen

Julianne Chung

Emil M. Constantinescu

Tobias Damm

Eric Darrigrand

Oleg Davydov

Johan De Villiers

Kristian Debrabant

Vittoria Demichelis

Weihua Deng

Josef Dick

Luca Dieci

Marco Donatelli

Cedric Effenberger

Yuli Eidelman

Lars Eldén

Jinyan Fan

Lihong Feng

Mahadevan Ganesh

Asghar Ghorbani

Oliver Gibary

Ron Goldman
Hector Gomez

Willy Govaerts

Ivan Graham

Andreas Griewank

Volker Grimm

Nicola Guglielmi

Hongbin Guo

Weihong Guo

Johnny Guzman

Mert Gürbüzbalaban

Stefan Güttel

Wolfgang Hackbusch

Thomas Hagstrom

Ernst Hairer

Harri Hakula

Xuli Han

Martin Hanke

Elias Helou

Johan Helsing

Holger Heumann

Nicholas Higham

Inmaculada Higueras

Martin Hutzenthaler

Johan Håstad

Zdzislaw Jackiewicz

Carlo Janna

Elias Jarlebring

Jae-Hun Jung

Mika Juntunen

Lars Karlsson

Linda Kaufman-Grabiner

Eamonn Keogh

Esther Klann

Peter E Kloeden

Othmar Koch

Plamen Koev

Efi Kokiopoulou

Richard Kowar

Rolf Krause

Natasa Krejic 
Ming-Jun Lai

Bishnu Lamichhane

Stig Larsson

Stephen Lau

Richard Lehoucq

Daniel Lesnic

Ren-Cang Li

Shijun Liao

Yongdo Lim

Thorsten Linss

Qiaohua Liu

Maria Maciel

Scott MacLachlan

Alexander Malyshev

Håkon Marthinsen

Gunnar Martinsson

Stefano Maset

Marie-Laurence Mazure

Robert McLachlan

William McLean

Gerard Medioni

Karl Meerbergen

Christian Mehl

Gerard Meurant

Ronald Mickens

Vladimir Mityushev

Juan Monterde

Julio Moro

James Nagy

Yuji Nakatsukasa

Andreas Neuenkirch

Hong Diep Nguyen

Harald Niederreiter

Touray Nikazad

Jan Nordström

Eugene O'Riordan

Peeter Oja

Sheehan Olver

Alexander Ostermann

Brynjulf Owren
Jose M. Peña

Rodrigo Platte

Helmut Podhaisky

Helmut Pottman

Weifeng Qiu

Ronny Ramlau

Joachim Rang

Nils Henrik Risebro

Mickael Robbe

Lucia Romani

Miroslav Rozloznik

Siegfried M. Rump

Paul Sablonnière

Miloud Sadkane

Ahmed Salam

Berkant Savas

Valeriu Savcenco

Achim Schaedle

Othmar Scherzer

Dominik M Schoetzau

Gabriela Schranz-Kirlinger

Simon Shaw

Qin Sheng

Alexandra Smirnova

Mark Spijker

Ernst Stephan

Hai-Wei Sun

Magnus Svärd

Lukas Szpruch

Gustaf Söderlind

Xue-Cheng Tai

Münevver Tezer Sezgin

Tianhai Tian

Francoise Tisseur

Stanimir Tomov

Ninoslav Truhar

Warwick Tucker

Miroslav Tuma

Stefan Turek

Andre Uschmajew 
Preface to BIT 53:4

825

Marnix Van Daele

Robert A van de Geijn

Martin Van Gijzen

Erik Van Vleck

Lieven Vandenberghe

Bart Vandereycken

Wim Vanroose

Stephen Vavasis

Martin Vohralik

Matthias Voigt
Junping Wang

David S Watkins

Yimin Wei

J.A.C. Weideman

Brendt Wohlberg

Jianlin Xia

Chuanju Xu

Chao Yang

Qianqian Yang

Antonella Zanna

Shangyou Zhang

Thank you all and see you later in BIT,

CAen Puke

Axel Ruhe

Springer 